\title{
The Nature of Space and of the Gravitational Fields Revealed by Recent Experimental Observations and Also Implicit in Quantum Field Theory
}

\author{
Jacob Schaff \\ Universidade Federal do Rio Grande do Sul (UFRGS), Instituto de Física, Porto Alegre, Brazil \\ Email: schaf@if.ufrgs.br
}

Received February 27, 2013; revised March 29, 2013; accepted April 26, 2013

Copyright (C) 2013 Jacob Schaff. This is an open access article distributed under the Creative Commons Attribution License, which permits unrestricted use, distribution, and reproduction in any medium, provided the original work is properly cited.

\begin{abstract}
The present work breaks the endless impasse of the current theories with space and gravitation, proposing a completely new conception in which the quantum space, ruling the propagarion of light and the inertial motion of matter, moves according to a velocity field consistent with the local main astronomical motions. This solution is clearly suggested by recent clear-cut experimental observations, achieved with the help of the GPS and also is implicit in the Quantum Field Theory (QFT) underlying the Standard Elementary Particle Model (SEPM). In a first part (Section II) it is shown that these recent experimental observations demonstrate that real space, the one that rules the propagation of light and the inertial motion of matter, is moving round each gravitational source according to a Keplerian velocity field consistent with the local main astronomical motions. This is the crucial experimental fundamentation of the spacedynamics that appropriately produces the observed gravitational dynamics on earth, in the solar system and also the galactic gravitational dynamics without the need of dark matter as well as all the observed effects of the gravitational fields on the propagation of light and on the rate of clocks. In a second part (Section III) it is shown how this spacedynamics arises within the context of the QFT underlying the SEPM. The QFT entails the idea that space is filled up with a scalar quantum field, a Bose-Einstein condensate of Higgs bosons. This Higgs condensate is a quantum fluid, responsible for giving mass to the elementary particles by the Higgs mechanism providing them with mechanical properties. This lets clear that the Higgs condensate plays the role of real quantum space that rules the propagation of light and the inertial motion of matter and is the ultimate reference for rest and for motion of matter and light. Therefore, on moving according to a Keplerian velocity field, this condensate causes the observed gravitational dynamics as well as all the other observed effects caused by the gravitational fields.
\end{abstract}

Keywords: Gravitation; Gravitational Physics; Gravitational Effects; Higgs Condensate; Quantum Space

\section{Introduction}

In his General Theory of Relativity (GR) [1] Einstein imputes the free-fall and the curved motions of matter within the gravitational fields to inertial motions within static curved spacetime geometry, caused by the gravitational sources. Einstein has set up the equation that describes the spacetime curvature within gravitational fields that predicts the orbital motions, perihelion precession, the gravitational time dilation, the gravitational redshift, the gravitational deflection of light, the excess time delay of electromagnetic signals within the solar system and many other effects.

However, now an increasing number of clear-cut experimental observations, achieved with the help of the tightly synchronized clocks of the Global Positioning System (GPS) [2-4], cannot be explained by the Theory of Relativity (TR). These experimental facts provide solid evidence that Einstein's static spacetime curvature is only an apparent explanation that does not disclose the true natures of space and of the gravitational fields. Besides this lurks an even much more serious menace. GR cannot account for the observed gravitational dynamics of galaxies and moreover provides no physical grounds for the accelerated expansion of the universe. In order to amend these flaws, the ideas of dark matter and dark energy have been introduced. However, despite the immense experimental and theoretical efforts, to present date nobody has idea what dark matter and dark energy 
exactly are.

The Quantum Field Theory (QFT), underlying the Standard Elementary Particle Model (SEPM), entails the idea that space is filled up with the Higgs condensate, a quantum fluid medium that provides mechanical properties to the elementary particles by the Higgs mechanism. This condensate is stable up to $10^{15} \mathrm{~K}$ the critical temperature of the electroweak interaction. QFT has been extremely successful in explaining electromagnetic, electroweak and the strong nuclear interactions. However, all efforts to explain the gravitational interaction failed. Quantum Gravity (QG) imputes the gravitational interaction to gravitational forces created by quantum exchange interaction mediated by gravitons that are massless spin 2 gauge particles. Gravitons do not conform to the superposition principle and therefore the gravitongraviton interaction leads to endless troubles because of the non-renormalisable divergences. Moreover, to present date the graviton is a hypothetical particle that never has been detected.

\section{The Nature of the Gravitational Fields}

This Section underscores the experimental observations, achieved during the last decade with the help of the GPS. These experimental facts, on one hand disprove fundamental assumptions of the current theories about the nature of space and the gravitational fields and on the other hand they disclose a completely different view. In this new view space, that rules the propagation of light and the inertial motion of matter, is a real quantum medium that moves according to a Keplerian velocity field round the astronomical bodies, thereby naturally inducing the observed gravitational dynamics as well as all the other observed effects, caused by the gravitational fields.

\subsection{Recent Measurements of the One Way Velocity of Light Break the Century Old Believe of the Intrinsic Isotropy and Constancy of Light}

Many recent highly precise and unmistakable experimental facts, achieved with the help of the tightly synchronized clocks of the GPS, put in check fundamental assumptions of the current theories about the nature of space and of gravitation. The atomic clocks on board of the 24 GPS satellites as well as on ground can be synchronized by the common view method to within $0.1 \mathrm{~ns}$ (time for light travel $3 \mathrm{~cm}$ ). With the help of these synchronized clocks the one-way velocity of electromagnetic (EM) signals (light) has been precisely measured. An especially clear-cut measurement has been achieved using the twin satellites of the Gravity Recovery and Climate Experiment (GRACE) [2]. These twin satellites move in the same sense at nearly $8 \mathrm{~km} / \mathrm{sec}$ along copla- nar and practically identical circular polar orbits at 500 $\mathrm{km}$ of altitude, separated from each other by $200 \mathrm{~km}$ and their positions being monitored by the GPS within $3 \mathrm{~cm}$. These satellites are equipped for many tasks. One of them is measuring microgravity effects. To this purpose they need to be provided with highly precise atomic clocks synchronized to better than $0.16 \mathrm{~ns}$.

The satellites continuously exchange EM signals between them in both senses. It is observed that the signal transit time from the leading satellite to the rear satellite corresponds to a shortening by more than $5 \mathrm{~m}$ (17 ns), while the signal transit time from the rear satellite to the leading satellite is lengthened by more than $5 \mathrm{~m}$ (17 ns). These discrepancies correspond in both cases exactly to the distance moved by the receiving satellite during the signal transit time and are consistent with backward signal anisotropy of nearly $8 \mathrm{~km} / \mathrm{sec}$ with respect to the satellites. This anisotropy is two orders of magnitude larger than the experimental precision of the experiment and by the first time breaks the century old believe of the intrinsic isotropy and constancy of the velocity of light. It also shows that the EM signal (light) has a well defined and isotropic North-South velocity $(c)$ within the geostatic non-rotating reference, the same with respect to which the satellites are moving at $8 \mathrm{~km} / \mathrm{sec}$.

This observation unambiguously proves that the space that rules the propagation of light is not moving along the North-South direction with respect to earth. The observed anisotropy is due to the motion of the satellites. This means that earth-based anisotropy experiments would show no North-South light anisotropy. The immediate consequence of this clear-cut experimental result is the absolute need of revising the interpretation of all the light anisotropy experiments performed in the past century.

The purpose of most Michelson light anisotropy experiments was measuring the anisotropy of light due to the orbital and cosmic motion of Earth. Systematically all these experiments obtained null results, which now must be interpreted as showing that Earth has no resultant velocity with respect to real space that rules the propagation of light. Obviously this can make a sense only if this space moves with Earth round the Sun, with the solar system round the galactic center etc. This is certainly an important result. However, what is much more impressive is the fact that this motion of space correctly and genuinely generates the observed gravitational dynamics and all the other observed effects, caused by the gravitational fields as shown in reference [5]. It however must be noted here that the aim of some Michelson light anisotropy experiments using highly precise interferometers rotating within the earth-based laboratories was measuring anisotropy with respect to the laboratory itself and all 
of them have detected small positive anisotropies of about $8 \mathrm{~km} / \mathrm{sec}$, constant the whole day and the whole year and direction fixed with respect to the laboratory. Figure 1 displays the most complete anisotropy results obtained by D. Miller in his late and nonstop measurements day and night [6].

These anisotropy results give evidence that space that rules the propagation of light is moving through the earth-based laboratories at nearly $8 \mathrm{~km} / \mathrm{sec}$. This motion cannot be along the North-South direction because this would go into conflict with the result of the above highly reliable anisotropy results obtained in the GRACE project. This motion can only be along the West-East direction.

Another recent experimental observation that also clearly conflicts with the constancy and isotropy o light came about during the implementation of set ups for Very Long Baseline Interferometry (VLBI) radio astronomy observations $[3,4]$. This interferometric method can improve the resolution of images by orders of magnitude. The condition is that reception of the signals from distant objects by the earth-based telescopes or antennas is synchronous. This can be achieved within 0.1 ns with the help of the GPS clocks. According to the TR, recaption of EM signals from distant pulsars by equidistant earthbased antennas should be synchronous. Nevertheless, contradicting the TR, observations showed that the wave front of a given pulsar signal reaches the foregoing antenna along the earth's orbital motion up to $4.2 \mu \mathrm{s}$ before the rear antenna. This discrepancy exceeds the time resolution of the experiment by four orders of magnitude. However, along directions transverse to the earth's orbital motion the arrival is observed to be synchronous. The reason for this relatively enormous discrepancy along the earth's orbital motion is explained straightforwardly by spacedynamics, please see reference [5].

\subsection{Absence of Effects of the Solar Gravitational Field on the GPS Clocks}

The 24 GPS satellites are moving round the earth in six equidistant circular 12 hour period orbits inclined 55 degrees with respect to the earth's equator. Each orbit contains 4 satellites, nearly equidistant from each other. The earth's rotation axis is inclined by 23 degrees with respect to the earth's orbital plane. Hence, the satellites having orbital plane closest to the Earth-Sun axis pass 6 hs at the side closer to the Sun and then $6 \mathrm{~h}$ at the part of the orbit more distant from the Sun.

The effect of the gravitational potential $(U)$ on the rate of clocks, predicted by GR, is given by

$$
T(r)=T_{o}\left[1-2 U / c^{2}\right]^{-1 / 2}
$$

Here $T_{o}$ is the time period in the absence of a gra-

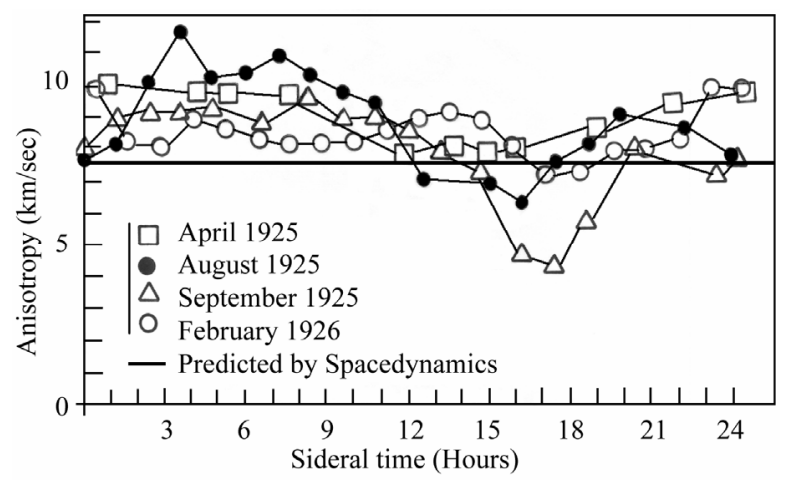

Figure 1. Ultimate light anisotropy data obtained by $D$. Miller. The small but systematic variations along the 24 hours may be due to spurious causes.

vitational potential and $c$ is the velocity of light. To first approximation this time dilation effect is proportional to $U / c^{2}$. Such gravitational time dilation effects have been well observed by atomic clocks within the earth's gravitational field and certainly would also be shown by clocks fixed within the solar gravitational field.

For the clocks in the GPS satellites having orbital plane closest to the Earth-Sun axis, GR predicts a total delay accumulated during the 6 hours of closest approach from the Sun of about 24 ns, which would be recovered during the subsequent 6 hours farthest from the Sun. The resulting 12 hour periodic sinusoidal variation in the time display of these clocks is more than two orders of magnitude larger than the stability of the clocks and, if present, would be very easily observed. Nevertheless, contradicting the prediction of GR, no sign of such variation is observed $[3,4]$. In fact observations show that the rate of the terrestrial and GPS clocks is ruled exclusively by the earth's gravitational field. GR cannot explain this absence because the gravitational potential is a scalar that depends on position but not on the orbital velocity of earth.

The absence of the time dilation effects of the solar gravitational field on the rate of the GPS clocks, which is predicted by the GR, but not observed, is the famous noon-midnight problem. This experimental observation has very drastic consequences on the current views about the nature of space and of gravitation. It moreover will be seen to fully corroborate the new interpretation of the light anisotropy experiments proposed in Section 2.1.

Some authors [7] suggest that the absence of effects of the solar gravitational field on the GPS clocks is due to the fact that these clocks together with earth are freefalling in the solar gravitational field. However, these same GPS clocks are also free-falling in the earth's gravitational field and notwithstanding are slowed by the earth's field by a quite significant and well observed amount. The assertion that a free falling clock is not affected by the gravitational field relies on the idea that 
gravitational time dilation is caused by acceleration. However, acceleration has been tested up to $10^{19} \mathrm{~m} / \mathrm{sec}^{2}$ by meson decay experiments in cyclotrons [8] with the clear-cut conclusion that accelerations do not cause time dilation.

Time dilation is well known to be caused by motion as evidenced by the well known increased lifetimes of speeding Muons and the redshift of the radiation emitted by speeding hydrogen atoms [9]. In the Special Theory of Relativity (STR) this time dilation effect is imputed to the relative velocity $\left(v_{r}\right)$ with respect to the observer. To first approximation this effect is proportional to $\left(v_{r} / c\right)^{2}$. Obviously the gravitational slowing of the atomic clocks on Earth cannot be due to relative velocity because these clocks rest with respect to the laboratory observer. However, if motions cause time dilation, why then does the orbital motion of Earth suppress the time dilation caused by the solar gravitational potential on the earthbased and GPS clocks? Absurdly in one case motion causes time dilation and in the other case it suppresses it. This contradiction lets clear that time dilation is not caused by relative velocity, nor is it caused by gravitational potential. Time dilation can only be caused by a velocity of a more fundamental nature.

The observed slowing of the atomic clocks on earth must be related with the observed small constant anisotropy of light of nearly $8 \mathrm{~km} / \mathrm{sec}$, both observed within the earthbased laboratories. Both these effects are proportional to $v^{2} / c^{2}=8^{2} / c^{2} \quad(c$ is in $\mathrm{km} / \mathrm{sec})$. Similarly the absence of effects due to the solar gravitational potential on the GPS clocks and all the clocks orbiting (with Earth) round the Sun must be related with the absence of light anisotropy due to the orbital motion of Earth as is well known. The mysterious implicit velocity $\left(V_{i}\right)$ that causes the small light anisotropy of nearly 8 $\mathrm{km} / \mathrm{sec}$ within the earthbased laboratories is the same that causes the observed small decrease of the clock rates on Earth. On the other hand, the orbital motion of Earth (30 $\mathrm{km} / \mathrm{sec}$ ) that suppresses the gravitational time dilation due to the solar gravitational field, also suppresses the light anisotropy on earth, caused by the implicit velocity $\left(V_{i}\right)$ due to the solar gravitational field. This singles out velocity as the unified cause of time dilation. It is the usual velocity in the case of clocks speeding in free space and it is this mysterious implicit velocity $V_{i}$ in the case of clocks fixed within gravitational fields.

The only way to provide physical reality to this implicit velocity $V_{i}$ is acknowledging that the real space, the one that rules the propagation of light and the inertial motion of matter and is the ultimate (locally absolute) reference for rest and for motions of matter, is itself moving round the Earth at $8 \mathrm{~km} / \mathrm{sec}$ on surface in the sense of the Moon's orbital motion as well as round the Sun according to a velocity field consistent with the earth's or- bital velocity $(30 \mathrm{~km} / \mathrm{sec})$ etc. This real space can be the ultimate (locally absolute) reference for rest and for motions of matter only if all the elementary particles and fields are in their essence perturbations of real quantum space itself. This will say that motions of the laboratory with respect to resting real quantum space or motions of real quantum space through the resting laboratory are perfectly equivalent and produce exactly the same physical effects.

In order to get mathematical rigor into the description, consider rectangular coordinate systems $[X, Y, Z]$ with origin fixed to the gravitational centers of earth, of the solar system, of the galaxy and directions of the axes fixed with respect to very distant objects, where the $Z$ axis points due north along the rotation axis of earth, of the solar system, of the galaxy. Moreover, let $(r, \theta, \phi)$ be the usual spherical coordinates and $\left(\boldsymbol{e}_{r}, \boldsymbol{e}_{\theta}, \boldsymbol{e}_{\phi}\right)$ unit vectors pointing along respectively the $r, \theta$ and $\phi$ spherical coordinates.

Obviously Earth cannot be kinematically privileged in detriment to all the other planets and astronomical bodies in general throughout the universe. Earth is not the only planet commoving with the real space in the velocity field round the Sun. All the planets must be commoving with the real quantum space, so that real quantum space must be moving according to a Keplerian velocity field round the Sun. In terms of the spherical coordinates, this Keplerian velocity field has the simple form:

$$
\boldsymbol{V}(r)=[\gamma M / r]^{1 / 2} \boldsymbol{e}_{\phi}
$$

This Keplerian velocity field has been shown in reference [5] to create the gravitational field. It is a cylindrical velocity field, the magnitude of the velocity for a given $r_{o}$ has the same value for all $\theta$ and $\phi$ and hence its magnitude is spherically symmetric. Such a Keplerian velocity field of real space must be circulating round each matter concentration throughout the universe, generating its gravitational field.

The natural orbital motions of astronomical bodies, which normally are all closely concentrated within the equatorial plane of the Keplerian velocity field (planetary satellites, solar system, galaxy etc.) are simply the combination of the circular motion of real space in the Keplerian velocity field together with their very slow inertial motion with respect to this moving real space. This will say that all these bodies very closely rest with respect to the moving real space, which explains why the light anisotropy experiments searching for light anisotropy due to the orbital and cosmic motions of earth gave null results. The circular orbital motions of the planets need not to be explained anymore because it is space itself that so moves. Only the very small deviations from the circular equatorial orbits that are due to the inertial 
motion of bodies with respect to the moving and distorting real space need to be explained. The vertical free fall is an extreme case in which the opposite implicit velocity $\left(V_{i}\right.$ of the body with respect to real space just compensates the Keplerian velocity.

Although this spacedynamics, which is evidenced by clear-cut experimental facts, seems to be a complicate choreography of space, it has been shown in reference [5] that this Keplerian velocity field of space is exactly the necessary one to correctly create the observed gravitational dynamics on earth, in the solar system and also generates correctly the galactic gravitational dynamics without the need of dark matter. It also correctly generates all the observed effects of the gravitational fields on the velocity of light and on the rate of clocks, including all the new effects recently discovered with the help of the GPS. It moreover simulates the non-Euclidean spacetime metric underlying Einstein's spacetime curvature and provides an antigravitation mechanism between oppositely rotating galaxies, which can explain the accelerated expansion of the universe.

If real quantum space rules the propagation of light (electromagnetic signals), then the effective velocity of light with respect to a laboratory moving at a velocity $v$ with respect to real space is given by:

$$
\boldsymbol{c}=\boldsymbol{c}+\boldsymbol{v}
$$

Hence within this laboratory the roundtrip time of a light pulse along a known distance is given by the usual well known mathematical expression:

$$
T(r)=T_{o}\left(1-v^{2} / c^{2}\right)^{-x}
$$

In this equation $T_{o}$ is the light roundtrip time along the same distance in a laboratory resting with respect to real quantum space, $x=1 / 2$ is for transverse go-return roundtrips and $x=1$ is for longitudinal go-return roundtrips (all intermediate values are possible). This light roundtrip is anisotropic (small) as shown by the light anisotropy experiments described in Section 2.1. The oscillation periods of the best time standards, used in atomic clocks are defined by electromagnetic (EM) oscillations of molecules or atoms. These oscillations too are go-return roundtrips of an EM signal along a certain distance that is totally equivalent to the light roundtrips and hence the EM oscillation period $T$ in the moving laboratory too is described by Equation (4). To first approximation the correction to the light roundtrip time as well as to the time standard roundtrip time $\Delta T$ is given by:

$$
\Delta T=x T_{o}\left(v^{2} / c^{2}\right)
$$

Therefore, the results of measurements, obtained with the method of light roundtrip and clocks, are constrained to give always the same value, independently from the velocity of the laboratory with respect to real space.
Within a laboratory fixed within a gravitational field, real space is moving through the laboratory according to the Keplerian velocity field Equation (2). Hence the fixed laboratory will be moving with respect to real space at the implicit velocity $\boldsymbol{V}_{i}(r)=-(\gamma M / r)^{1 / 2} \boldsymbol{e}_{\phi}$. This velocity is implicit because it is not velocity of the laboratory with respect to a usual reference, but of space with respect to the resting laboratory. In this case, the velocity of a light signal within the fixed laboratory will be given by:

$$
\boldsymbol{c}=\boldsymbol{c}+(\gamma M / r)^{1 / 2} \boldsymbol{e}_{\phi}
$$

Hence, in Equation (3) we set $v^{2}=V_{i}^{2}=\gamma M / r=U$ so that the light roundtrip time as well as the time standard must be corrected by:

$$
\Delta T(r)=x T_{o}\left(V_{i}^{2} / c^{2}\right)=x T_{o}\left(U / c^{2}\right)
$$

Atomic clocks on earth have effectively shown exactly this gravitational time dilation and certainly these clocks fixed within the solar system too would show the correction due to the solar velocity (gravitational) field given by Equation (2). On the other hand, for a clock moving in a direct circular equatorial orbit the velocity with respect to real quantum space is:

$$
\boldsymbol{v}=\boldsymbol{V}(r)+\boldsymbol{V}_{i}=(\gamma M / r)^{1 / 2} \boldsymbol{e}_{\phi}-(\gamma M / r)^{1 / 2} \boldsymbol{e}_{\phi}=0
$$

Hence, for clocks in direct circular equatorial orbits the gravitational time dilation vanishes as indeed observed for the GPS clocks moving with earth round the Sun.

For clocks moving along polar orbits the time dilation is 2 times larger than for clocks resting at the same altitude [see Equation (7)]. In particular, the GPS satellites move along circular orbits at $2.02 \times 10^{4} \mathrm{~km}$ of altitude, inclined 55 degrees with respect to the equator and hence their clocks have velocity components with respect to real space $v_{o}(1-\cos \alpha)$ along $-\phi$ and $v_{o} \sin \alpha$ along $\pm \theta$, where $v_{o}=3.87 \mathrm{~km} / \mathrm{sec}$ and $\alpha$ is the angle of their orbital velocity $v_{o}$ with respect to the equator or parallels. In reference [5] the author has shown that the rate of these clocks would be $4.5 \times 10^{-10} \mathrm{sec} / \mathrm{sec}$ faster than the rate of clocks on ground. Hence, in order to the GPS clocks to run synchronous with identical clocks on ground, they must be slowed by this value before launch. This is closely the preset that NASA implements in the rate of the GPS clocks before launch.

It may be important to recall here that in reference [5] it is shown that spacedynamics defined by Equation (2) correctly produces the free fall of bodies on earth, the gravitational dynamics within the solar system as well as the observed gravitational dynamics of the stars within the galactic disk, without the need of dark matter. It also suggests an antigravitational mechanism between oppositely rotating galaxies that may explain the accelerated 
expansion of the universe. It finally correctly produces the observed light anisotropies, the observed gravitational time dilation, the absence of gravitational time dilation of the solar field on the GPS clocks, the excess time delay of radar signal roundtrips within the solar system, the observed non-synchronous arrival of the pulsar wave fronts to earthbased antennas along the orbital motion of earth and the synchronous arrival along the transverse direction, the observed gravitational deflection of light by the Sun, the observed anomalous red-blue spectral shifts of radiation from binary stars and binary galaxies, the necessary slowing of the GPS clock rates before launch, the perihelion advance in elliptic orbits and many other effects of the gravitational fields.

\section{The Quantum Nature of Space Implicit in the Quantum Field Theory}

Obviously no classical spatial medium has the required physical properties to rule the propagation of light and the inertial motion of matter, let alone the observed gravitational dynamics of the astronomical systems. Only a very powerful and highly stable quantum fluid medium comes into consideration. The aim of this Section 3 is to disclose how the quantum space dynamics creating the gravitational fields emerges within the context of the Quantum Field Theory (QFT) underlying the Standard Elementary Particle Model (SEPM). This spacedynamics will be regarded as a macroscopic manifestation of the Higgs mechanism. QFT has extensively been guided by the well known phenomenologies of the usual quantum fluids, especially by the superconducting condensates [10]. It may be important to underscore for the reader the most important features of the superconducting condensates underlying the macroscopic manifestations of the Meissner effect in superconductors that may help understanding the macroscopic manifestation of the Higgs mechanism.

\subsection{Some Important Hinds from Superconducting Condensates and Superfluids}

Usual quantum fluids are Bose-Einstein (BE) condensates of bosonic particles. In spite of being infinitely deformable and perfectly inviscid for steady state motions, quantum fluids possess a peculiar quantum phase stiffness that enables them to propagate phase perturbations at very high velocity and to actively resist any changes of their actual state of motion. In superconductors BE condensation becomes possible only with pairing of conduction electrons near the Fermi level into Cooper pairs with zero spin and electric charge $2 e$. After condensation the Cooper pairs tunnel throughout the volume of the condensate and become completely entangled and indistin- guishable. The charged superconducting condensate is described by the complex order parameter $\psi=\psi(r) \mathrm{e}^{\mathrm{i} \theta}$, where $\psi$ is the amplitude, $|\psi|^{2}$ is the local condensate density and $\theta$ is the phase.

BE condensation occurs at low temperature because of the quantum phase correlation between the particle wavefunctions, which leads to a long-range phase coherence. This phase correlation gives rise to a negative potential energy (bonding) term that increases with the condensate density as $|\psi|^{2}$. Another positive potential energy (antibonding) term arises from repulsive core interaction between the particles that increases as $|\psi|^{4}$. However, the coefficient of the $|\psi|^{2}$ term is significantly larger than that of the $|\psi|^{4}$ term. Therefore the minimum of the effective potential energy occurs not for $\psi=0$, as usual, but for a finite value of $\psi$. This characterizes the so called Mexican hat potential. When the temperature of the system becomes sufficiently low, the phase correlation between the particle wave-functions enforces spontaneous breaking of the global $U(1)$ gauge symmetry by which the particle wavefunctions assume all a same arbitrary phase. In superconductors $1 \mathrm{meV}$ of energy is liberated per Cooper pair during BE condensation. In this quantum coherent state, the long range order parameter $\psi$ represents the resting condition of the condensate.

Local motion of the quantum fluid with respect to the overall fluid is excited when the local phase of part of the condensate is displaced with respect to the overall phase of the order parameter. The condensate flows along the phase gradient $(\nabla \theta)$ with velocity proportional to the magnitude of the gradient. Phase gradients are caused by an interacting field. In superconductors the vector potential $\boldsymbol{A}$ associated with magnetic field is well known to cause a local phase shift of the superconducting order parameter. While a constant phase gradient leads to uniform flow of the condensate, a phase gradient changing with time corresponds to accelerated motion of the condensate. Any change of the amplitude or phase of the order parameter involves energy as described in the Ginsburg-Landau theory [11].

Another very fundamental property of quantum fluids is the fact that the phase correlation of the order parameter between the volume elements of the quantum fluid does not act in the sense of stabilizing the position of one volume element with respect to the others (solidification) however in the sense of preventing all changes in the actual state of motion of the quantum fluid throughout. This phase correlation between the volume elements provides the quantum fluid with a characteristic dynamical stiffness, despite flowing and continuously deforming like a usual fluid. This gives it the ability of actively resisting against all changes of the state of motion and is responsible for its perfect persistence (absence of all internal dissipation). Active opposition to variation of 
phase means that quantum fluids preserve the already existing phase displacements. This is why any motion in a quantum fluid, once excited, becomes persistent. For instance, if a current is excited in a superconducting coil, it will flow forever and only can be stopped by an opposing electromotive force. Quantum fluids are intrinsically perfectly conservative systems.

Stationary circulation fields along closed loops, caused by a vector potential field, contain a locked-in phase displacement. Single valuedness requires that the total phase displacements $\theta$ round the loops be an integer number (n) of $2 \pi$.

$$
\theta=\frac{2 \pi}{\phi_{o}} \oint \boldsymbol{A} \cdot \mathrm{d} \boldsymbol{I}=n 2 \pi
$$

where $\phi_{o}$ is the unit of magnetic flux in the flux tube. This leads to intrinsic quantization of the perturbation. The origin of this intrinsic quantization is different from the usual quantization due to particle confinement by potential barriers. In type II superconductors, magnetic flux quantization occurs due to confinement of the magnetic field into quantized fluxons by the Meissner effect at a microscopic scale. Quantized Abrikosov supercurrent vortices [12] round the fluxons complete screening of the magnetic field within the London penetration depth. The screening supercurrents must not be conceived as classical motion of individual Cooper pairs but as a local drift velocity field of the superconducting condensate itself. The screening current intensity falls off exponentially with distance from the fluxon.

Superconductivity and magnetic fields are intrinsically incompatible with each other. The vector potential associated to the magnetic field causes phase displacements that destroy the phase coherence of the electron wave functions and thus the pairing correlation. This breaks the Cooper pairs and tends to locally reestablish gauge symmetry. Superconductivity and magnetic field coexist by sharing space. The superconducting condensate tends to get rid of the magnetic field by developing screening currents and a Lorentz (reaction) force field that thrusts the magnetic field out from the superconductor or compresses it into the smallest volume possible. In its turn the magnetic field displaces the phase of the superconducting order parameter by its associated vector potential, thereby destroying locally superconductivity, recovering the local gauge symmetry and creating a screening interface layer that is a velocity field (vortex) of the condensate. In neutral quantum fluids or superfluids a totally similar phenomenology takes place. This antagonism between condensate and deleterious phase distortions seems to be a feature common to all quantum fluids.

For macroscopic use of superconductivity several important new aspects come into concern. The Meissner flux expulsion is the macroscopic counterpart of the microscopic Meissner effect confining the magnetic field into flux tubes. Expulsion of large bundles of magnetic fluxons involves a macroscopic Lorentz (reaction) force field. Under a sufficiently dense fluxon distribution, besides repulsion between the Abrikosov vortices, the residual collective vector potential field together with the curl-free part excites macroscopic screening currents (velocity field of the condensate) round the bundle, resulting in a collective Lorentz (reaction) force field along the gradient of the fluxon density. The curl-free part of the vector potential is well known to cause phase shifts of the order parameter $[13,14]$. This Lorentz force field thrusts the magnetic flux along the flux density gradient and normally expels it out from the superconductor. However, in non-homogeneous superconductors, in which the amplitude of the superconducting order parameter has internally weaker regions (lower transition temperature), the Lorentz (reaction) force field may compress the fluxons into these weaker superconducting regions. The compressed magnetic flux weakens even more the local superconductivity, which leads to the well known high field paramagnetic Meissner effect [15].

\subsection{The Higgs Condensate as the Quantum Space}

Usual Bose-Einstein (BE) condensates are all very frail. Contrarily the Quantum Space (QS) or quantum vacuum, propagating the electromagnetic waves and responsible for the gravitational dynamics, must be an extremely powerful quantum fluid medium, stable up to extremely high temperatures. The QFT underlying the Standard Elementary Particle Model entails the idea that space is filled up with the scalar Higgs field that is an $S U(2)$ doublet, a spinor field with two complex components. The Higgs field was introduced after verifying that the electroweak interaction is short-range and that the vector gauge bosons $W^{+}, W^{-}$and $Z$, mediating it, are massive. Spontaneous breaking of the global $U(1)$ gauge symmetry at $10^{15} \mathrm{~K}$ leads to $\mathrm{BE}$ condensation of the Higgs bosons.

Analogously as the superconducting condensate confines the electromagnetic field making it short-range and giving mass to the photons, the Higgs condensate, pervading all of space, confines the electroweak field, making it short-range and giving mass to the $W^{+}, W^{-}$ and $Z$ bosons. Likewise the superconducting condensate, the Higgs condensate too is a real quantum fluid, which however is stable up to $10^{15} \mathrm{~K}$, the critical temperature of the electroweak nuclear interaction. The Higgs condensate also provides inertial mass to the quarks and leptons by an indirect Yukawa like coupling to the Higgs condensate. Thereby quarks and leptons become con- 
fined and get mass. Moreover all their parameters become quantized. The Higgs mechanism hence rules the inertial motion of matter. It is responsible for the mechanical properties of the elementary particles and also rules the propagation of light. This lets clear that the Higgs condensate plays the role of the quantum space, as asserted in Section 2.2 and hence is locally the ultimate reference for rest and for motion of matter. This will say that, on moving itself according to a differential velocity field, it will cause an inertial dynamics of matter exactly of the kind observed within the gravitational fields.

In the relativistic gauge theory, all the elementary particles are originally massless gauge particles. They acquire mass by interacting with the Higgs condensate. This however only shows that, on interacting with the Higgs condensate, the particles exhibit inertial mass. It in no way tells what inertial mass really is. The inertial mass can only be a manifestation of a property of the Higgs condensate itself, the quantum space responsible for the mechanical properties of the particles. It is a manifestation of the phase correlation responsible for the spontaneous breaking of the gauge symmetry of the Higgs field as well as for the conservation of the actual state of motion of the quantum space. Likewise the excitations in the superconducting condensate are persistent; excitations in the Higgs condensate too are persistent. From this viewpoint inertial mass and the conservation of linear and angular momenta of bodies is simply consequence of the persistence and conservation of all motions in the Higgs condensate. Any motion, once excited in the condensate, becomes persistent.

For a particle propagating at velocity lower than the velocity of light, phase correlation also becomes effective along the longitudinal direction. In the QFT this corresponds to the particles acquiring a longitudinal degree of freedom and hence mass. In practice, the Higgs mechanism and mass acquisition must be concomitant with the creation of the particles. The QFT provides us with a theoretical procedure showing that, on connecting to the Higgs condensate, a field looses global gauge symmetry and gets mass. There however is a problem. The Higgs condensate is really a quantum fluid in which no parts are distinguishable. Therefore coupling of the particles to the Higgs condensate becomes possible only if the involved particles are in their essence phase perturbations (dynamical states) of the Higgs condensate itself. This confirms that the inertial mass of the particles is effectively a manifestation of the phase correlation of the Higgs condensate. In other words, inertia and conservation of linear and angular momenta are simply persistent modes of the Higgs condensate.

The Higgs condensate is to the electroweak and strong nuclear fields what the superconducting condensate is to magnetic fields. The Higgs mechanism is the quantum space analog of the Meissner effect in superconductors. As seen above, the Meissner effect excludes electromagnetic fields from the superconductor thereby breaking their gauge symmetry and turning them short range. This makes the photons massive within superconductors. The Higgs mechanism excludes the electroweak fields from the Higgs condensate (from quantum space) by confining it into a minimum distance of about $10^{-19} \mathrm{~m}$. This turns it into an extremely short-range interaction and aggregates large rest mass to the $W^{+}, W^{-}$and $Z$ gauge vector bosons of the electroweak interaction. The structure of the Higgs condensate is different from that of the superconducting condensate. However, both are quantum fluids and this must be the reason of the close similarity of their phenomenologies. Evidently this close resemblance can not end here.

Likewise the superconducting condensate is antagonistic to magnetic fields, the Higgs condensate is antagonistic to the electroweak fields but not to the electromagnetic and gravitational fields. While the electroweak and strong nuclear fields are deleterious to the Higgs condensate, electromagnetic and gravitational fields are not. Likewise the Meissner effect is related to the phase shifts of the order parameter by the magnetic vector potential, the Higgs mechanism may arise from phase shifts of the Higgs order parameter by the vector potential analogs associated with the electroweak nuclear field. Local phase displacements of the order parameter of a quantum fluid by vector potentials excite motion of the condensate (screening currents). The superconducting condensate contains electric charges and therefore screens or confines magnetic fields. The Higgs condensate contains electroweak hypercharges and therefore its motions screen and confine the electroweak fields. It however contains no electric charge and hence it cannot screen the electromagnetic field.

The Higgs condensate also does not screen gravitational fields. According to reference [5], the gravitational interaction is not an exchange interaction. Therefore the affair of the interaction range and of mass of the gravitons simply does not arise. According to reference [5] gravitation is essentially the result of the usual inertial motion of matter with respect to quantum space (Higgs condensate) that is moving in the ordinary three dimensions round astronomical bodies according to the Keplerian velocity field $\boldsymbol{V}(r)=(\gamma M / r)^{1 / 2} \boldsymbol{e}_{\phi}$ consistent with the local main astronomical motions. Since the Higgs condensate is the ultimate reference for rest and or motion of matter, its motions according to the Keplerian velocity field induce the observed gravitational dynamics. The fact that this Keplerian velocity field decreases with distance according to $(r)^{-1 / 2}$ must be related with geometry and the phase correlation of the Higgs condensate. 
This $(r)^{-1 / 2}$ dependence also is the fundamental feature of the velocity field that creates a centrifugal force field not outward, but toward the gravitational center [5]. In conclusion, the electroweak and strong nuclear fields become extremely short range and normally produce no relevant macroscopic effects. On the other hand, electromagnetic interactions that are long range rarely become relevant in a macroscopic scale because of mutual screening of positively and negatively charged particles. The only interaction that effectively remains long range is the gravitational interaction and hence it is completely dominant in an astronomical scale.

The resemblance between the microscopic Meissner effect and the microscopic Higgs mechanism is certainly auspicious and this may go on in their macroscopic manifestations. Likewise the Meissner effect, the Higgs mechanism too must have a macroscopic counterpart. In a superconductor the macroscopic screening currents, induced by the residual vector potential of a large bundle of magnetic fluxons, develop a Lorentz (reaction) force field that thrusts the magnetic flux along the gradient of the flux density out from the superconductor or into weaker superconducting regions [15]. Analogously, a macroscopic velocity field of the Higgs condensate may be induced by the vector potential analogs of the electroweak field of a large concentration of matter. Such macroscopic velocity fields too must generate a macroscopic force field along the gradient of the matter density distribution thrusting and compressing matter toward regions where the order parameter of the condensate has already been weakened by a large matter concentration. According to reference [5], the screening velocity field of the Higgs condensate round spherically symmetric matter distributions is the Keplerian velocity field of the quantum space and the corresponding force field is the gravitational field that in fact is a centrifugal force field toward the gravitational center.

This quantum space dynamics bridges together the idea of QFT, according to which a spatial medium, the Higgs condensate rules the inertial behavior of matter and Einstein's idea of the equivalence of inertial and gravitational effects. However, in the view of this spacedynamics, gravitation is not due to inertial motion within Einstein's static curved spacetime. It also is not the result of gravitational forces created by the quantum exchange interaction mediated by gravitons, as proposed in Quantum Gravity. It is really due to the inertial motion (propagation) of matter and light within real quantum space that is moving in the ordinary three dimensions round gravitational sources according to a Keplerian velocity field consistent with the local main astronomical motions. This same spacedynamics also naturally and correctly produces in terms of genuine physical effects all the other observed effects, caused by the gravitational fields, on the propagation of light and on the rate of the clocks.

\section{Spatial and Time Invariance of the Laws of Physics throughout the Universe}

Many observations indicate that the laws of physics are the same throughout the universe and also along time. In reference [5] it is shown that the actual motions of the astronomical bodies throughout the universe essentially trace out very closely the motion of the quantum space itself creating the gravitational fields. This must have been so since the formation of the first galaxies. This will say that all these astronomical bodies do very closely rest with respect to the local moving quantum space (Higgs condensate) that rules the propagation of light and the inertial motion of matter. This universality of the laws of physics is a straightforward consequence of the fact that the physical phenomena taking place on earth and in all these worlds evolve locally under closely the same kinematical circumstances with respect to the local quantum space that is the ultimate reference for rest and for motion of matter. This spatial invariance of the laws of physics however is clearly not the same thing as Lorentz invariance. Lorentz invariance only would be unambiguously proved if the laws of physics were observed to be exactly the same in very different inertial references at the same place.

The superconducting condensate is physically anchored to the superconducting material. It is not Lorentz invariant because it has a preferred reference that is the superconducting material. The Higgs condensate, the quantum space has no such preferred reference. However, viewed that all the elementary particles are in their essence mere local phase perturbations of this Higgs condensate and get their mechanical properties from it, they cannot represent a reference for the motion of the Higgs condensate. Hence, this moving condensate clearly is the ultimate reference for rest and for motion of matter. Therefore along its motions this quantum space carries with it all the ultimate and locally absolute reference for rest and for motion of matter as well as for all the material phenomena. This will say that the moving Higgs condensate, the quantum space is automatically itself the local preferred reference for rest and or motion of matter, so that its motion is a gauge invariant that is completely innocuous to the local physical phenomena. This automatically assures the sameness of the laws of physics throughout the universe as observed. Now several experimental observations provide indubitable prove (please see Section 2) that within a gravitational field this condensate (quantum space) moves according to a Keplerian velocity field, consistent with the local main astronomical motions, this way creating the gravitational fields. Hence, within gravitational fields, the preferred reference 
is a different one at each point of space. Each planet of the solar system, each star in the galactic disk and each galaxy represent very closely the local preferred reference because all these bodies very closely rest with respect to the local moving quantum space. On observing that physical events on Mars or on very distant galaxies evolve the same way as on earth, one naively could conclude that the laws of physics are Lorentz invariant, that is, are not affected by the apparent relative velocity of these bodies. From the viewpoint of the present work, these laws are the same not because of Lorentz invariance, however because the kinematical circumstances with respect to the local moving quantum space, the locally absolute reference for rest and motion of matter, are the same in all these worlds.

\section{Some Important Conclusions}

The central idea of the present work (see also reference [5]) is that real space, the one that rules the propagation of light and the inertial motion of matter, moves within the gravitational fields round each gravitational source according to a Keplerian velocity field. This Keplerian velocity field of real space appropriately creates the observed gravitational dynamics on Earth, in the solar system as well as the galactic gravitational dynamics without the need of dark matter and all the other observed effects, caused by the gravitational fields on the propagation of light and on the rate of clocks. It is shown that severeal recent clear-cut experimental observations, achieved with the help of the GPS, reveal exactly this spacedynamics and that moreover the QFT, underlying the SEPM, contains the physical elements that may generate this spacedynamics. This resolves the impasse in the current theories about the nature of space and of gravitation.

\section{REFERENCES}

[1] H. A. Lorentz, A. Einstein, H. Minkowski and H. Weyl, "The Principle of Relativity," Dover Publications Inc., New York.

[2] R. R. Hatch, Physics Essays, Vol. 20, 2007, p. 83. doi:10.4006/1.3073811

[3] R. R. Hatch, GPS Solutions, Vol. 8, 2004, p. 67. doi:10.1007/s10291-004-0092-8

[4] R. R. Hatch, Foundations of Physics, Vol. 34, 2004, p. 1725 .

[5] J. Schaff, Journal of Modern Physics, Vol. 3, 2012, pp. 714-749. doi:10.4236/jmp.2012.38097

[6] D. C. Miller, Review of Modern Physics, Vol. 5, 1933, p. 203. doi:10.1103/RevModPhys.5.203

[7] Neil Ashby, Private Communication.

[8] H. Bailey, K. Borer, F. Combley, H. Drumm and F. Krienen, Nature, Vol. 268, 1977, pp. 301-305. doi: $10.1038 / 268301 \mathrm{a} 0$

[9] H. E. Ives and G. R. Stilwell, Journal of the Optical Society of America, Vol. 28, 1938, p. 215. doi:10.1364/JOSA.28.000215

[10] L. Dixon, "From Superconductors to Supercolliders." slac.stanford.edu/pubs/beamline/26/1/26-1-dixon.pdf

[11] V. L. Ginzburg and L. D. Landau, Pisma v Zhurnal Eksperimentalnoi i Teoreticheskoi Fiziki, Vol. 20, 1950, p. 1064.

[12] Ai. A. Abrikosov, Soviet Physics Journal of Experimental and Theoretical Physics, Vol. 5, 1957, p. 1174.

[13] Y. Aharonov and D. Bohm, Physical Review, Vol. 115, 1959, p. 485. doi:10.1103/PhysRev.115.485

[14] R. K. Varma, A. M. Punithavelu and S. B. Banerjee, Physics Letters A, Vol. 303, 2002, p. 114. doi:10.1016/S0375-9601(02)01223-9

[15] F. T. Dias, P. Pureur, P. Rodrigues Jr. and X. Obradors, Physical Review B, Vol. 70, 2004, Article ID: 224519. doi:10.1209/0295-5075/89/37005 